\title{
ON THE BIHOMOGENEITY PROBLEM OF KNASTER
}

\author{
KRYSTYNA KUPERBERG
}

\begin{abstract}
The author constructs a locally connected, homogeneous, finitedimensional, compact, metric space which is not bihomogeneous, thus providing a compact counterexample to a problem posed by B. Knaster around 1921.
\end{abstract}

\section{INTRODUCTION}

A topological space $X$ is said to be homogeneous if for every two points $p$ and $q$ in $X$ there exists a homeomorphism $h: X \rightarrow X$ such that $h(p)=q . X$ is said to be bihomogeneous if for every two points $p$ and $q$ in $X$ there exists a homeomorphism $h: X \rightarrow X$ such that $h(p)=q$ and $h(q)=p$. Around 1921 , B. Knaster asked the question of whether every homogeneous space is bihomogeneous, and shortly after that C. Kuratowski (see [6]) described an example of a non-locally-compact, homogeneous subset of the plane, which is not bihomogeneous. In 1930, D. van Danzig asked whether homogeneity implies bihomogeneity for continua; see [10]. A locally compact, homogeneous, nonbihomogeneous, metric space was found by H. Cook in the early 1980 s; see [3].

This paper contains an example of a seven-dimensional, homogeneous, nonbihomogeneous, locally connected, compact metric space. G. S. Ungar proved that certain homogeneity type properties imply local connectedness; see [9]. However, [5] and this paper show that a locally connected homogeneous continuum may lack some stronger but still very simple homogeneity properties.

The author would like to thank Piotr Minc for his cooperation and valuable suggestions. One of his remarks is that, using similar methods, one can obtain a simpler, although not locally connected, example of a homogeneous, nonbihomogeneous continuum.

\section{Preliminaries}

All spaces considered in this paper are metric and all maps are continuous. By $S^{n}, E^{n}, B^{n}$, and $\bar{B}^{n}$ we mean the $n$-dimensional sphere, the Euclidean $n$-space, the $n$-dimensional open ball, and the $n$-dimensional closed ball respectively.

Received by the editors February 2, 1988.

1980 Mathematics Subject Classification (1985 Revision). Primary 54F20; Secondary 57M10. 
Let $P$ and $Q$ be two disjoint, closed subsets in a compact space $X$, and let $g: P \rightarrow Q$ be a homeomorphism. Let $\sim$ be an equivalence relation on $X$ such that $p \sim q$ iff $p=q$, or if $p \in P$ and $q \in Q$ then $g(p)=q$, or if $q \in P$ and $p \in Q$ then $g(q)=p$. The space of equivalence classes with the quotient topology will be denoted by $X / g$.

In our applications of homology theory, we use either the singular or Čech homology groups with integral coefficients. For basic concepts of homotopy theory we refer the reader to [4].

Throughout this paper, $M$ will denote the universal Menger curve as described in R. D. Anderson's paper [1, p. 321]. $M$ is a subset of the cube $\left\{(x, y, z) \in E^{3}: x, y, z \in[0,1]\right\}$ such that the intersection of $M$ with each of the faces of the cube is homeomorphic to Sierpinski's plane curve.

In [1], Anderson proved that $M$ is homogeneous, and that every 1-dimensional continuum with no local cut points and no open subsets embeddable in the plane is homeomorphic to $M$. Furthermore, from results in [1 and 2], it follows that if $U$ is an open connected subset of $M$, and $p, q \in U$, then there exists a homeomorphism $h: M \rightarrow M$ such that $h(p)=q$, and $h(v)=v$ for $v \in M-U$.

In [5], the authors employ the fact that continua which are Cartesian products with one or more factors homeomorphic to $M$ admit few homeomorphisms. A similar idea is used here in the form of Lemmas 1 and 2 whose proofs are analogous to those of Theorems 2 and 1 in [5].

Lemma 1. Let $X=X_{1} \times X_{2}$, where $X_{i}$ is homeomorphic to $M$ for $i=1,2$. Let $U_{i} \subset X_{i}$ be a connected open set for $i=1$, 2. If $\varphi: U_{1} \times U_{2} \rightarrow X$ is an open embedding, then $\varphi=\varphi_{1} \times \varphi_{2}$, where either (1) $\varphi_{1}: U_{1} \rightarrow X_{1}$ and $\varphi_{2}: U_{2} \rightarrow X_{2}$, or (2) $\varphi_{1}: U_{1} \rightarrow X_{2}$ and $\varphi_{2}: U_{2} \rightarrow X_{1}$.

Proof. Let $\pi_{i}: X \rightarrow X_{i}$ be the projection. Suppose that $\left(u, v_{1}\right)$ and $\left(u, v_{2}\right)$ are two distinct points in $U_{1} \times U_{2}$. Let $\varphi\left(\left(u, v_{1}\right)\right)=\left(x_{1}, y_{1}\right)$ and $\varphi\left(\left(u, v_{2}\right)\right)=$ $\left(x_{2}, y_{2}\right)$. Suppose that $x_{1} \neq x_{2}$ and $y_{1} \neq y_{2}$. Let $V_{1} \subset X_{1}$ and $V_{2} \subset X_{2}$ be such that $V_{1} \times V_{2}$ is a neighborhood of $\left(x_{1}, y_{1}\right)$ contained in $\varphi\left(U_{1} \times U_{2}\right)$.

There exists a nonsingular loop $f: S^{1} \rightarrow U_{1}$ such that $u \in f\left(S^{1}\right)$, and if $f_{i}: S^{1} \rightarrow X$ is defined by $f_{i}(s)=\left(f(s), v_{i}\right)$ for $i=1,2$, then $\varphi \circ f_{1}\left(S^{1}\right) \in$ $V_{1} \times V_{2}$, and for $i=1,2$, we have $\pi_{i} \circ \varphi \circ f_{1}\left(S^{1}\right) \cap \pi_{i} \circ \varphi \circ f_{2}\left(S^{1}\right)=\varnothing$. Since $f_{1}\left(S^{1}\right)$ is a retract of $U_{1} \times U_{2}$, then $\varphi \circ f_{1}\left(S^{1}\right)$ is a retract of $\varphi\left(U_{1} \times U_{2}\right)$, and hence a retract of $V_{1} \times V_{2}$. Therefore $\varphi \circ f_{1}$ is essential, which implies that at least one of the maps $\pi_{i} \circ \varphi \circ f_{1}$ is essential. Suppose that $\pi_{1} \circ \varphi \circ f_{1}$ is essential. Since $U_{2}$ is arcwise connected, then $f_{1}$ and $f_{2}$ are homotopic, and hence $\pi_{1} \circ \varphi \circ f_{1}$ and $\pi_{1} \circ \varphi \circ f_{2}$ are homotopic. However, no two essential and disjoint loops in the Menger curve are homotopic. Therefore either $x_{1}=x_{2}$ or $y_{1}=y_{2}$.

If $x_{1}=x_{2}$, then for any $v_{3} \in U_{2}$ we have $\varphi\left(\left(u, v_{3}\right)\right)=\left(x_{1}, y_{3}\right)$. Since a similar fact can be shown for points in $U_{1} \times U_{2}$ with equal second coordinate, 
then $\varphi=\varphi_{1} \times \varphi_{2}$, where $\varphi_{1}: U_{1} \rightarrow X_{1}$ and $\varphi_{2}: U_{2} \rightarrow X_{2}$.

If $y_{1}=y_{2}$ then $\varphi=\varphi_{1} \times \varphi_{2}$, where $\varphi_{1}: U_{1} \rightarrow X_{2}$ and $\varphi_{2}: U_{2} \rightarrow X_{1}$.

Lemma 2. Let $X=X_{1} \times X_{2}$, where $X_{1}$ is homeomorphic to $M \times M$, and $X_{2}$ is a continuum whose every point has a closed neighborhood which is an absolute retract. For $i=1,2$, let $U_{i} \subset X_{i}$ be an open set and let $U_{2}$ be connected. If $\varphi: U_{1} \times U_{2} \rightarrow X$ is an open embedding, then for every $u \in U_{1}$ there exists an $x \in X_{1}$ such that $\varphi\left(\{u\} \times U_{2}\right) \subset\{x\} \times X_{2}$.

Proof. Suppose that $\left(u, v_{1}\right)$ and $\left(u, v_{2}\right)$ are points in $U_{1} \times U_{2}$. Let $\varphi\left(\left(u, v_{1}\right)\right)$ $=\left(x_{1}, y_{1}\right)$ and let $\varphi\left(\left(u, v_{2}\right)\right)=\left(x_{2}, y_{2}\right)$. Suppose that $x_{1} \neq x_{2}$. Let $V_{1} \subset X_{1}$ and $V_{2} \subset X_{2}$ be such that $V_{1} \times V_{2}$ is a neighborhood of $\left(x_{1}, y_{1}\right)$ contained in $\varphi\left(U_{1} \times U_{2}\right)$, and $V_{2}$ is an absolute retract. Let $\pi_{1}: X \rightarrow X_{1}$ be the projection.

There exists an embedding $f: S^{1} \times S^{1} \rightarrow U_{1}$ such that $u \in f\left(S^{1} \times S^{1}\right)$, $f\left(S^{1} \times S^{1}\right)$ is a retract of $X_{1}$, and if $f_{i}: S^{1} \times S^{1} \rightarrow X$ is defined by $f_{i}(s)=$ $\left(f(s), v_{i}\right)$ for $i=1,2$, then $\pi_{1} \circ \varphi \circ f_{1}\left(S^{1} \times S^{1}\right) \cap \pi_{1} \circ \varphi \circ f_{2}\left(S^{1} \times S^{1}\right)=\varnothing$, and $\varphi \circ f_{1}\left(S^{1} \times S^{1}\right) \subset V_{1} \times V_{2}$. Note that $f_{1}$ and $f_{2}$ are homotopic. Since $f_{1}\left(S^{1} \times S^{1}\right)$ is a retract of $U_{1} \times U_{2}, \varphi \circ f_{1}\left(S^{1} \times S^{1}\right)$ is a retract of $\varphi\left(U_{1} \times U_{2}\right)$, and hence $\varphi \circ f_{1}\left(S^{1} \times S^{1}\right)$ is a retract of $V_{1} \times V_{2}$. Let $g: X_{1} \rightarrow X$ be an embedding defined by $g(p)=\left(p, y_{1}\right)$. Using the Čech homology and the induced homomorphism, we have $0 \neq\left(\varphi \circ f_{1}\right)_{*}(a)=\left(g \circ \pi_{1} \circ \varphi \circ f_{1}\right)_{*}(a)=\left(g \circ \pi_{1} \circ \varphi \circ f_{2}\right)_{*}(a)$, where $a$ is a generator of $\mathrm{H}_{2}\left(S^{1} \times S^{1}\right)$.

Therefore, there are two 2-dimensional nontrivial homologous Čech cycles with disjoint carriers in $X_{1}$. By [7, p. 246], the dimension of $X_{1}$ is greater than 2 , which is a contradiction.

\section{THE TWISTED PRODUCTS}

Denote by $(r, \theta, z)$ the cylindrical coordinates of a point in $E^{3}$. Let $\mu$ be an embedding of the Menger curve $M$ in $E^{3}$ defined by $\mu(x, y, z)=$ $(r, \theta, z)$, where $r=x+1, \theta=\frac{2 \pi}{9} y$, and $z=z$, for every $(x, y, z) \in M$. Let $f(r, \theta, z)=\left(r, \theta+\frac{2 \pi}{9}, z\right)$ be the rotation about the $z$-axis through the angle of $\frac{2 \pi}{9}$. Put $A_{0}=\mu(M), A_{k}=f^{(k)}\left(A_{0}\right)$, where $f^{(k)}$ is the $k$ th iteration of $f$, and put $A=\bigcup_{i=0}^{8} A_{i}$.

Clearly, $A$ is invariant under $f$, and $f_{1}=\left.f\right|_{A}$ is a periodic homeomorphism of $A$ onto itself. By [1], $A$ is homeomorphic to $M$. Cylindrical coordinates $(r, \theta, z)$ will be used to denote a point in $A$, and Cartesian coordinates $(\bar{x}, \bar{y}, \bar{z})$ will be used to denote a point in $M$. If $p=(a, m) \in A \times M$ is a point, where $a=(r, \theta, z)$ and $m=(\bar{x}, \bar{y}, \bar{z})$, then $p$ may be denoted by $(r, \theta, z, \bar{x}, \bar{y}, \bar{z})$.

For every $\alpha \in[0,1]$, put $M_{\alpha}=\{(\bar{x}, \bar{y}, \bar{z}) \in M: \bar{z}=\alpha\}$. Let $g_{1}: M_{1} \rightarrow M_{0}$ be the homeomorphism taking $(\bar{x}, \bar{y}, 1)$ onto $(\bar{x}, \bar{y}, 0)$. Let $g_{2}: A \times M_{1} \rightarrow$ $A \times M_{0}$ be defined by $g_{2}(a, m)=\left(f_{1}(a), g_{1}(m)\right)$. Define $B$ as the quotient space $(A \times M) / g_{2}$. 
Thus the continuum $B$, a twisted product of $A$ and $M$, is obtained from the Cartesian product $A \times M$ by pasting the "top" $A \times M_{1}$ to the "bottom" $A \times M_{0}$. Points in $B$ will be denoted in the same manner as the corresponding points in $A \times M$ for which $\bar{z} \neq 1$.

Define $f_{2}: B \rightarrow B$ by $f_{2}(a, m)=\left(f_{1}(a), m\right)$ for $a \in A$ and $m \in M-M_{1}$. Clearly, $f_{2}$ is a periodic homeomorphism of period 9 .

Lemma 3. For every two points $p, q \in B$ there exists a homeomorphism $h: B \rightarrow$ $B$ such that $h(p)=q$ and $h \circ f_{2}=f_{2} \circ h$.

Proof. First, we shall show that for every two points $p=\left(r_{p}, \theta_{p}, x_{p}, \bar{x}_{p}, \bar{y}_{p}\right.$, $\left.\bar{z}_{p}\right)$ and $q=\left(r_{q}, \theta_{q}, x_{q}, \bar{x}_{q}, \bar{y}_{q}, \bar{z}_{q}\right)$ there exists a homeomorphism $h_{1}: B \rightarrow B$ such that $h_{1}(p)=\left(r_{p}, \theta_{p}, z_{p}, \bar{x}_{q}, \bar{y}_{q}, \bar{z}_{q}\right)$ and $h_{1} \circ f_{2}=f_{2} \circ h_{1}$.

Let $M / g_{1}$ be the space homeomorphic to $M$ obtained from $M$ by identifying, in a similar fashion as above, the point $(\bar{x}, \bar{y}, 1)$ with the point $(\bar{x}, \bar{y}, 0)$ for $\bar{x}, \bar{y} \in[0,1]$. For $\alpha \in[0,1)$, denote by $\widetilde{M}_{\alpha}$ the subset of $M / g_{1}$ corresponding to $M_{\alpha} \subset M$. The point in $M / g_{1}$ corresponding to the point $(\bar{x}, \bar{y}, \bar{z}) \in M$, where $\bar{z} \neq 1$, will be denoted by $(\bar{x}, \bar{y}, \bar{z})$.

For every $\alpha \in[0,1)$, put $B_{\alpha}=\left\{(a, m) \in B: m \in M_{\alpha}\right\}$. The map $\Psi_{\alpha}: B-$ $B_{\alpha} \rightarrow A \times\left(M / g_{1}-\widetilde{M}_{\alpha}\right)$ defined by

$$
\Psi_{\alpha}(r, \theta, z, \bar{x}, \bar{y}, \bar{z})= \begin{cases}((r, \theta, z),(\bar{x}, \bar{y}, \bar{z})) & \text { if } 0 \leq \bar{z}<\alpha, \\ \left(\left(r, \theta+\frac{2 \pi}{9}, z\right),(\bar{x}, \bar{y}, \bar{z})\right) & \text { if } \alpha<\bar{z}<1,\end{cases}
$$

is a homeomorphism.

There exists a number $\alpha_{0}$ and there exists a connected open subset $U \subset$ $M / g_{1}$ containing $\left(\bar{x}_{p}, \bar{y}_{p}, \bar{z}_{p}\right)$ and $\left(\bar{x}_{q}, \bar{y}_{q}, \bar{z}_{q}\right)$ such that $U \cap \widetilde{M}_{\alpha_{0}}=\varnothing$. There exists a homeomorphism $k_{1}: M / g_{1} \rightarrow M / g_{1}$ taking $\left(\bar{x}_{p}, \bar{y}_{p}, \bar{z}_{p}\right)$ onto $\left(\bar{x}_{q}, \bar{y}_{q}, \bar{z}_{q}\right)$, and not moving points outside $U$. Let $\bar{k}_{1}: A \times\left(M / g_{1}-\widetilde{M}_{\alpha_{0}}\right) \rightarrow$ $A \times\left(M / g_{1}-\widetilde{M}_{\alpha_{0}}\right)$ be such that

$$
\bar{k}_{1}((r, \theta, z),(\bar{x}, \bar{y}, \bar{z}))=\left((r, \theta, z), k_{1}(\bar{x}, \bar{y}, \bar{z})\right),
$$

and define $\bar{h}_{1}: B \rightarrow B$ by setting

$$
\bar{h}_{1}(v)= \begin{cases}\Psi_{\alpha_{0}}^{-1} \circ \bar{k}_{1} \circ \Psi_{\alpha_{0}}(v) & \text { if } v \notin B_{\alpha_{0}}, \\ v & \text { if } v \in B_{\alpha_{0}} .\end{cases}
$$

Hence,

$$
\bar{h}_{1}(p)=\Psi_{\alpha_{0}}^{-1} \circ k_{1} \circ \Psi_{\alpha_{0}}(p)=\left(r_{p}, \theta_{p}+\varepsilon \frac{2 \pi}{9}, z_{p}, \bar{x}_{q}, \bar{y}_{q}, \bar{z}_{q}\right),
$$

where $\varepsilon \in\{0,1,-1\}$. Put

$$
h_{1}(v)= \begin{cases}\bar{h}_{1}(v) & \text { if } \varepsilon=0 \\ f_{2}^{-1} \circ \bar{h}_{1}(v) & \text { if } \varepsilon=1 \\ f_{2} \circ \bar{h}_{1}(v) & \text { if } \varepsilon=-1\end{cases}
$$


For any $v=(r, \theta, z, \bar{x}, \bar{y}, \bar{z}) \in B, h_{1}(v)=\left(\left(r, \theta+\delta \frac{2 \pi}{9}, z\right), k_{1}(\bar{x}, \bar{y}, \bar{z})\right)$, where $\delta \in\{0,1,-1\}$. Hence $h_{1} \circ f_{2}(v)=\left(\left(r, \theta+(\delta+1) \frac{2 \pi}{9}, z\right), k_{1}(\bar{x}, \bar{y}, \bar{z})\right)=$ $f_{2} \circ h_{1}(v)$.

Next, we shall show that there exists a homeomorphism $h_{2}: B \rightarrow B$ such that $h_{2}\left(r_{p}, \theta_{p}, z_{p}, \bar{x}_{q}, \bar{y}_{q}, \bar{z}_{q}\right)=q$, and $h_{2} \circ f_{2}=f_{2} \circ h_{2}$.

If $f_{2}^{(i)}\left(r_{p}, \theta_{p}, z_{p}\right)=\left(r_{q}, \theta_{q}, x_{q}\right)$ for some $i$, then set $h_{2}=f_{2}^{(i)}$. Otherwise, there is an open connected set $U \subset A$ containing $\left(r_{p}, \theta_{p}, z_{p}\right)$ and $\left(r_{q}, \theta_{q}, z_{q}\right)$, and such that the sets $U, f(U), \ldots, f^{(8)}(U)$ are pairwise disjoint. There is a homeomorphism $k_{2}: A \rightarrow A$ which is the identity outside $U$ taking $\left(r_{p}, \theta_{p}, z_{p}\right)$ onto $\left(r_{q}, \theta_{q}, z_{q}\right)$. Define $h_{2}$ by

$$
h_{2}(v)= \begin{cases}\left(f^{(i)} \circ k_{2} \circ\left(f^{(i)}\right)^{-1}(a), m\right) & \text { if } a \in f^{(i)}(U), \\ v & \text { if } a \notin \bigcup_{j=1}^{9} f^{(j)}(U),\end{cases}
$$

where $v=(a, m)$ and $i=1, \ldots, 9$.

Clearly, $h_{2} \circ f_{2}=f_{2} \circ h_{2}$.

Finally, put $h=h_{2} \circ h_{1}$.

Let $n$ be a positive integer, and let $N$ be an $n$-manifold with nonempty boundary such that $\partial N=N_{0} \cup N_{1}$, where $N_{0} \cap N_{1}=\varnothing$, both $N_{0}$ and $N_{1}$ are closed, and there exists a homeomorphism $g_{3}: N_{1} \rightarrow N_{0}$. Let $g_{4}: B \times N_{1} \rightarrow$ $B \times N_{0}$ be such that $g_{4}(b, s)=\left(f_{2}^{(3)}(b), g_{3}(s)\right)$, where $f_{2}^{(3)}$ is the third iteration of $f_{2}$. Define $Z_{N}$ as the quotient space $(B \times N) / g_{4}$.

Points in $Z_{N}$ will be denoted in the same way as the corresponding points in the Cartesian products $B \times N$ or $A \times M \times N$. Specifically, if $p \in Z_{N}$, then $p=(b, s)$, where $b \in B$ and $s \in N-N_{1}$, or $p=(a, m, s)$, where $a \in A, m \in M-M_{1}$, and $s \in N-N_{1}$, or $p=(r, \theta, z, \bar{x}, \bar{y}, \bar{z}, s)$, where $(r, \theta, z) \in A,(\bar{x}, \bar{y}, \bar{z}) \in M-M_{1}$, and $s \in N-N_{1}$.

Lemma 4. $Z_{N}$ is homogeneous.

Proof. Let $p=\left(b_{p}, s_{p}\right)$ and $q=\left(b_{q}, s_{q}\right)$ be two points in $Z_{N}$. To show that there exists a homeomorphism $h: Z_{N} \rightarrow Z_{N}$ taking $p$ onto $q$, it is enough to show that there are homeomorphisms $h_{1}, h_{2}: Z_{N} \rightarrow Z_{N}$ such that $h_{1}(p)=$ $\left(b_{p}, s_{q}\right)$ and $h_{2}\left(b_{p}, s_{q}\right)=q$.

Let $U$ be a neighborhood of $b_{p}$ in $B$ such that $U, f_{2}^{(3)}(U)$, and $f_{2}^{(6)}(U)$ are pairwise disjoint. The set $W=\left\{(b, s) \in Z_{N}: b \in \bigcup_{i=3,6,9} f_{2}^{(i)}(U)\right\}$ is homeomorphic to $U \times Q$, where $Q$ is an $n$-manifold; in fact, $Q$ is a union of three copies of $N$. For any two points $d_{1}$ and $d_{2}$ in $Q$, there exists a homeomorphism $k: Q \rightarrow Q$ isotopic to the identity such that $k\left(d_{1}\right)=d_{2}$. Using the isotopy and the Cartesian product structure of $W$, it is easy to obtain the homeomorphism $h_{1}: Z_{N} \rightarrow Z_{N}$ with $h_{1}\left(b_{p}, s_{p}\right)=\left(b_{p}, s_{q}\right)$ and $h_{1}(v)=v$ for $v \notin W$.

By Lemma 3, there exists a homeomorphism $\bar{h}: B \rightarrow B$ such that $\bar{h}\left(b_{p}\right)=b_{q}$ and $\bar{h} \circ f_{2}=f_{2} \circ \bar{h}$. In particular, $\bar{h} \circ f_{2}^{(3)}=f_{2}^{(3)} \circ \bar{h}$. Hence, $h_{2}: Z_{N} \rightarrow Z_{N}$, 
where $h_{2}(b, s)=(\bar{h}(b), s)$, is well defined and $h_{2}\left(b_{p}, s_{q}\right)=q$.

Let $p=\left(a_{0}, m_{0}\right)$ be a point in $B$. The sets $A_{p}$ and $M_{p}$ are defined by

$$
\begin{aligned}
& A_{p}=\left\{(a, m) \in B: m=m_{0}\right\}, \\
& M_{p}=\left\{(a, m) \in B: a=f_{1}^{(i)}\left(a_{0}\right), \text { where } i=1, \ldots, 9\right\} .
\end{aligned}
$$

Similarly, if $p=\left(b_{0}, s_{0}\right) \in Z_{N}$, then the sets $B_{p}$ and $N_{p}$ are defined by

$$
\begin{aligned}
& B_{p}=\left\{(b, s) \in Z_{N}: s=s_{0}\right\}, \\
& N_{p}=\left\{(b, s) \in Z_{N}: b=f_{2}^{(i)}\left(b_{0}\right), \text { where } i=3,6,9\right\} .
\end{aligned}
$$

Each of the sets $A_{p}, M_{p}, B_{p}$, and $N_{p}$ will be called a fiber.

Lemma 5. If $h: B \rightarrow B$ is a homeomorphism, then either (1) $h\left(A_{p}\right)=A_{h(p)}$ and $h\left(M_{p}\right)=M_{h(p)}$ for all $p \in B$, or $(2) h\left(A_{p}\right)=M_{h(p)}$ and $h\left(M_{p}\right)=A_{h(p)}$ for all $p \in B$.

Proof. Every point in $B$ has a closed neighborhood in the form of a Cartesian product $X_{1} \times X_{2}$, where $X_{1}$ is a subset of $A$ homeomorphic to $M$, and by means of a homeomorphism similar to the homeomorphism $\Psi_{\alpha}$ of Lemma 3, $X_{2}$ is a subset of $M / g_{1}$ homeomorphic to $M$. Moreover, for every $x_{1} \in X_{1}$ and $x_{2} \in X_{2}$, each of the sets $\left\{x_{1}\right\} \times X_{2}$ and $X_{1} \times\left\{x_{2}\right\}$ is contained in a fiber $A_{p}$ or $M_{p}$. Since $B$ is compact, there exists a finite collection $\left\{V_{1}, \ldots, V_{k}\right\}$ of these neighborhoods such that $B=\bigcup_{i=1}^{k} \operatorname{Int}\left(V_{i}\right)$. Similarly, every point in $B$ has arbitrarily small open neighborhoods in the form $U_{1} \times U_{2}$, where for $i=1,2, U_{i}$ is homeomorphic to a connected subset of $M$. Let $\left\{W_{1}, \ldots, W_{l}\right\}$ be a finite collection of these neighborhoods covering $B$, and such that for each $j=1, \ldots, l$, there is an $i$ such that $h\left(W_{j}\right) \subset V_{i}$. By Lemma 1 , if $p \in B$, then for each $j=1, \ldots, l$, there is an $i$ such that $h\left(A_{p} \cap W_{j}\right) \subset A_{h(p)} \cap V_{i} \subset A_{h(p)}$ or $h\left(A_{p} \cap W_{j}\right) \subset M_{h(p)} \cap V_{i} \subset M_{h(p)}$. Hence $h\left(A_{p}\right) \subset A_{h(p)}$ or $h\left(A_{p}\right) \subset M_{h(p)}$. Since $B$ is connected, then if $h\left(A_{p}\right) \subset A_{h(p)}\left[h\left(A_{p}\right) \subset M_{h(p)}\right]$ for one point $p \in B$, then $h\left(A_{p}\right) \subset A_{h(p)}\left[h\left(A_{p}\right) \subset M_{h(p)}\right]$ for every $p \in B$. A similar statement holds for $M_{p}$. Since $h$ is one-to-one, we have $h\left(A_{p}\right)=A_{h(p)}$ and $h\left(M_{p}\right)=M_{h(p)}$ for $p \in B$, or we have $h\left(A_{p}\right)=M_{h(p)}$ and $h\left(M_{p}\right)=A_{h(p)}$ for $p \in B$.

Let $p \in B$ be a point. Denote by $O_{p}$ the orbit of $p$ under $f_{2}$, i.e., $O_{p}=$ $A_{p} \cap M_{p}=\left\{p, f_{2}(p), \ldots, f_{2}^{(8)}(p)\right\}$. The following lemma is an immediate consequence of Lemma 5 .

Lemma 6. If $h: B \rightarrow B$ is a homeomorphism, then $h\left(O_{p}\right)=O_{h(p)}$ for every $p \in B$.

Lemma 7. Let $p_{i}=\left(1, \frac{2 \pi i}{9}, 0,0,0,0\right) \in B$ for $i=0, \ldots, 8$. If $h: B \rightarrow B$ is a homeomorphism such that $h\left(p_{0}\right)=p_{i_{0}}$ and $h\left(p_{1}\right)=p_{i_{1}}$, then $h\left(p_{j}\right)=$ $p_{\left[i_{0}+j\left(i_{1}-i_{0}\right)\right] \bmod 9}$.

Proof. Let $L_{i}$ be the arc $\left\{(1, \theta, 0,0,0,0) \in B: \frac{2 \pi i}{9} \leq \theta \leq \frac{2 \pi(i+1)}{9}\right\}$, where $i=0, \ldots, 8$. The set $L=\bigcup_{i=0}^{8} L_{i}$ is a simple closed curve invariant under 
$f_{2}$. By Lemma 6, $\bigcup_{i=1}^{9} f_{2}^{(i)} \circ h\left(L_{0}\right) \subset h(L)$. Furthermore, for $i=0, \ldots, 8$, the end points of the arc $f_{2}^{(i)} \circ h\left(L_{0}\right)$ are the points $p_{\left(i_{0}+i\right) \bmod 9}$ and $p_{\left(i_{1}+i\right) \bmod 9}$. Therefore, since $h(L)$ is a simple closed curve, we have $\bigcup_{i=1}^{9} f_{2}^{(i)} \circ h\left(L_{0}\right)=h(L)$. Hence, the ends of the arc $h\left(L_{j}\right)$ are $p_{\left[i_{0}+j\left(i_{1}-i_{0}\right)\right] \bmod 9}$ and $p_{\left[i_{0}+(j+1)\left(i_{1}-i_{0}\right)\right] \bmod 9}$. Thus $h\left(p_{j}\right)=p_{\left[i_{0}+j\left(i_{1}-i_{0}\right)\right] \bmod 9}$.

Lemma 8. If $h: Z_{N} \rightarrow Z_{N}$ is a homeomorphism, then $h\left(N_{p}\right)=N_{h(p)}$ for every $p \in Z_{N}$.

Proof. Every point in $Z_{N}$ has a closed neighborhood in the form of a Cartesian product $X_{1} \times X_{2}$, where $X_{1}$ is homeomorphic to $M \times M$ and $X_{2}$ is homeomorphic to a closed ball $\bar{B}^{n}$. We may assume that if $p\left(x_{1}, x_{2}\right) \in X_{1} \times X_{2}$, then $\left\{x_{1}\right\} \times X_{2} \subset N_{p}$ and $X_{1} \times\left\{x_{2}\right\} \subset B_{p}$. Since $Z_{N}$ is compact, there exists a finite collection $\left\{V_{1}, \ldots, V_{k}\right\}$ of these neighborhoods such that $Z_{N}=\bigcup_{i=1}^{k} \operatorname{Int}\left(V_{i}\right)$. Similarly, every point in $Z_{N}$ has arbitrarily small neighborhoods in the form $U_{1} \times U_{2}$, where $U_{1}$ is homeomorphic to an open subset in $M \times M$, and $U_{2}$ is homeomorphic to an open ball $B^{n}$. Let $\left\{W_{1}, \ldots, W_{l}\right\}$ be a finite collection of these neighborhoods covering $Z_{N}$ such that for every $j=1, \ldots, l$, there is an $i$ such that $h\left(W_{j}\right) \subset V_{i}$. By Lemma 2 , if $p \in Z_{N}$, then for every $j=1, \ldots, l$, there is an $i$ such that $h\left(N_{p} \cap W_{j}\right) \subset N_{h(p)} \cap V_{i} \subset N_{h(p)}$. Since $N_{p}$ is connected, then $h\left(N_{p}\right) \subset N_{h(p)}$.

Now, we will define a continuum $C$ by putting $C=Z_{N}$, where $N=[0,1]$, $N_{1}=\{1\}, N_{0}=\{0\}$, and $g_{3}(1)=0$. Notice that $C=\{(b, s): b \in B$ and $s \in[0,1)\}$. Consider $B$ to be the subset $\{(b, s) \in C: s=0\}$ of $C$.

Let $(\rho, \alpha)$ denote the polar coordinates in the plane. Assume that $S^{1}=$ $\left\{(\rho, \alpha) \in E^{2}: \rho=1\right.$ and $\left.\alpha \in[0,2 \pi)\right\}$. Let $\Gamma: C \rightarrow S^{1}$ be defined by

$$
\Gamma(r, \theta, z, \bar{x}, \bar{y}, \bar{z}, s)=\left(1,\left(\theta+\frac{2 \pi}{9} \bar{z}+\frac{2 \pi}{3} s\right) \bmod 2 \pi\right) .
$$

Clearly, $\Gamma$ is continuous.

Lemma 9. For every point $p \in C,\left.\Gamma\right|_{N_{p}}: N_{p} \rightarrow S^{1}$ is a homeomorphism.

Proof. If $p=\left(r_{p}, \theta_{p}, z_{p}, \bar{x}_{p}, \bar{y}_{p}, \bar{z}_{p}, s_{p}\right)$, then

$$
N_{p}=\left\{\left(r_{p}, \theta_{p}+\frac{2 \pi}{3} \varepsilon, z_{p}, \bar{x}_{p}, \bar{y}_{p}, \bar{z}_{p}, s\right): \varepsilon \in\{0,1,2\} \text { and } s \in[0,1)\right\} .
$$

$\Gamma\left(\left(r_{p}, \theta_{p}+\frac{2 \pi}{3} \varepsilon, z_{p}, \bar{x}_{p}, \bar{y}_{p}, \bar{z}_{p}, s\right)\right)=\left(1,\left(\theta_{p}+\frac{2 \pi}{3} \varepsilon+\frac{2 \pi}{9} \bar{z}_{p}+\frac{2 \pi}{3} s\right) \bmod 2 \pi\right)$. Clearly, $\Gamma$ is one-to-one and therefore $\Gamma$ is a homeomorphism.

Consider $H_{1}\left(S^{1}\right)$ to be the additive group of integers. For every $p \in C$, denote by $a_{p}$ the generator of $H_{1}\left(N_{p}\right)$ such that $\left(\left.\Gamma\right|_{N_{p}}\right)_{*}\left(a_{p}\right)=1$. Just the first homology group determines an orientation on $S^{1}$ and on each fiber $N_{p}$. 
Definition. A homeomorphism $h: C \rightarrow C$ is said to be orientation preserving [reversing] if for every $p \in C,\left.h\right|_{N_{p}}$ is orientation preserving [reversing].

Lemma 10. If $k: C \rightarrow C$ is a map and if for every $p \in C$ there exists a $p^{\prime} \in C$ such that $k\left(N_{p}\right) \subset N_{p^{\prime}}$, then for any two points $p_{1}$ and $p_{2}$ in $C$, we have $\left(\left.\Gamma \circ k\right|_{N_{p_{1}}}\right)_{*}\left(a_{p_{1}}\right)=\left(\left.\Gamma \circ k\right|_{N_{p_{2}}}\right)_{*}\left(a_{p_{2}}\right)$.

Proof. There exists a finite open cover $\left\{V_{i}\right\}$ of $C$ such that each $V_{i}$ is homeomorphic to $\left(V_{i} \cap B\right) \times S^{1}$, and such that if $V_{i} \cap V_{j} \neq \varnothing$, then the two Cartesian product structures coming from $V_{i}$ and $V_{j}$ are compatible. Hence, any two simple closed curves $N_{p_{1}}$ and $N_{p_{2}}$ bound a singular annulus. Therefore $\left(\left.\Gamma \circ k\right|_{N_{p_{1}}}\right)_{*}\left(a_{p_{1}}\right)=\left(\left.\Gamma \circ k\right|_{N_{p_{2}}}\right)_{*}\left(a_{p_{2}}\right)$.

Lemma 11 follows immediately from Lemma 10.

Lemma 11. If $h: C \rightarrow C$ is a homeomorphism, then $h$ is orientation preserving or $h$ is orientation reversing.

Lemma 12. Let $p_{i}=\left(1, \frac{2 \pi i}{9}, 0,0,0,0,0\right) \in C$, where $i=0, \ldots, 8$. Let $h: C \rightarrow C$ be a homeomorphism such that $h(B)=B$. If $h\left(N_{p_{0}}\right)=N_{p_{1}}$ and $h\left(N_{p_{1}}\right)=N_{p_{0}}$, then $h$ is orientation reversing.

Proof. Since $h\left(\left\{p_{0}, p_{3}, p_{6}\right\}\right)=\left\{p_{1}, p_{4}, p_{7}\right\}$, and $h\left(\left\{p_{1}, p_{4}, p_{7}\right\}\right)=\left\{p_{0}, p_{3}\right.$, $\left.p_{6}\right\}$, we have $h\left(p_{0}\right)=p_{i_{0}}$, where $i_{0} \bmod 3=1$, and $h\left(p_{1}\right)=p_{i_{1}}$, where $i_{1} \bmod 3=0$. By Lemma $7, h\left(p_{j}\right)=p_{\left[i_{0}+j\left(i_{1}-i_{0}\right)\right] \bmod 9}$ for $j=0, \ldots, 8$. Therefore $h\left(p_{3}\right)=p_{\left(i_{0}+6\right) \bmod 9}$ and $h\left(p_{6}\right)=p_{\left(i_{0}+3\right) \bmod 9}$ which implies that $h$ is orientation reversing.

\section{THE EXAMPLE}

Assume the following notation:

$$
\begin{aligned}
J^{n} & =\left\{\left(x_{1}, \ldots, x_{n}\right) \in E^{n}: x_{1} \in[0,1]\right\}, \\
J_{0}^{n-1} & =\left\{\left(x_{1}, \ldots, x_{n}\right) \in E^{n}: x_{1}=0\right\}, \\
J_{1}^{n-1} & =\left\{\left(x_{1}, \ldots, x_{n}\right) \in E^{n}: x_{1}=1\right\} .
\end{aligned}
$$

For $i<n$, consider $E^{i}$ to be the subset of $E^{n}$ for which $x_{i+1}=\cdots=x_{n}=0$.

Let $T$ be the Möbius strip with $\partial T=T_{1}$, and let $T_{0}$ be the middle simple closed curve of $T$. Consider $T$ to be the mapping cylinder of $\gamma: T_{1} \rightarrow T_{0}$, where $\gamma$ is a map of degree 2. Since there is a piecewise linear embedding of $T$ in $E^{3}$, we may assume that $T$ is a piecewise linear subset of $J^{4}$ with $T_{i}=T \cap J_{i}^{3}$ for $i=0,1$. Let $\Sigma T_{i}$ be the suspension of $T_{i}$. Denote by $\Sigma T$ the mapping cylinder of the suspension of $\gamma$. Again, assume that $\Sigma T$ is a piecewise linear subset of $J^{5}$ with $\Sigma T_{i}=\Sigma T \cap J_{i}^{4}$ for $i=0,1$. Let $V$ be a regular neighborhood of $\Sigma T$ in $J^{5}$ such that for $i=0,1, V_{i}=V \cap J_{i}^{4}$ is a regular neighborhood of $\Sigma T_{i}$. Let $V^{\prime}, V^{\prime \prime}, V_{0}^{\prime}, V_{0}^{\prime \prime}$, and $V_{1}^{\prime}, V_{1}^{\prime \prime}$ be two copies of $V, V_{0}$, 
and $V_{1}$ respectively. Denote by $\sigma: \partial V^{\prime}-\operatorname{Int}\left(V_{0}^{\prime} \cup V_{1}^{\prime}\right) \rightarrow \partial V^{\prime \prime}-\operatorname{Int}\left(V_{0}^{\prime \prime} \cup V_{1}^{\prime \prime}\right)$, $\sigma_{0}: \partial V_{0}^{\prime} \rightarrow \partial V_{0}^{\prime \prime}$, and $\sigma_{1}: \partial V_{1}^{\prime} \rightarrow \partial V_{1}^{\prime \prime}$ the homeomorphisms corresponding to the identity homeomorphisms. Assume that $V$ has an orientation compatible with the orientation of $E^{5}$, and assume that each $V_{i}$ has an orientation induced by the orientation of $V$. Let $\bar{\gamma}: V_{1} \rightarrow V_{0}$ be an orientation reversing homeomorphism, and let $\bar{\gamma}^{\prime}: V_{1}^{\prime} \rightarrow V_{0}^{\prime}$ and $\bar{\gamma}^{\prime \prime}: V_{1}^{\prime \prime} \rightarrow V_{0}^{\prime \prime}$ be the homeomorphisms corresponding to $\bar{\gamma}$. Let $G=\left(V^{\prime} \cup V^{\prime \prime}\right) / \sigma$. Note that $\partial G=G_{0} \cup G_{1}$, where $G_{0}=\left(V_{0}^{\prime} \cup V_{0}^{\prime \prime}\right) / \sigma_{0}$ and $G_{1}=\left(V_{1}^{\prime} \cup V_{1}^{\prime \prime}\right) / \sigma_{1}$ are disjoint sets, each homeomorphic to $S^{2} \times S^{2}$. The homeomorphisms $\bar{\gamma}^{\prime}$ and $\bar{\gamma}^{\prime \prime}$ yield a homeomorphism $\hat{\gamma}: G_{1} \rightarrow G_{0}$.

Denote by $D$ the continuum obtained by putting $D=Z_{N}$, with $N=G$ and $g_{3}=\hat{\gamma}$, where $g_{4}$ is the map appearing in the definition of $Z_{N}$ given in $\S 2$. Each fiber $N_{p}$ of $D$ is an orientable 5-manifold $F$ which is a union of three copies of $G$ intersecting along the boundary components.

Let $L$ be a properly embedded arc in $G$ with end points $q$ and $g_{3}(q)$ on $G_{1}$ and $G_{0}$, respectively. There exists a retraction $r: G \rightarrow L$ such that $r^{-1}(q)=$ $G_{1}, r^{-1}\left(g_{3}(q)\right)=G_{0}$. We can write $F=G^{0} \cup G^{1} \cup G^{2}$ with $G_{1}^{i}=G_{0}^{(i+1) \bmod 3}$ for $i=0,1,2$, where $G^{i}, G_{0}^{i}$, and $G_{1}^{i}$ are copies of $G, G_{0}$, and $G_{1}$, respectively. Let $L_{i} \subset G^{i}$ be an arc corresponding to the $\operatorname{arc} L \subset G$. Put $K=L_{0} \cup L_{1} \cup L_{2}$. Note that $K$ is a retract of $F$. Let $\bar{r}: F \rightarrow K$ be a retraction such that for $i=0,1,2,\left.\bar{r}\right|_{G^{i}}: G^{i} \rightarrow L_{i}$ is the retraction corresponding to $r$. Notice that $\bar{r}$ induces an isomorphism of the first homology groups $\bar{r}_{*}: H_{1}(F) \rightarrow H_{1}(K)$.

Let $\tau: \widetilde{F} \rightarrow F$ be a covering map such that $\tau^{-1}(K)$ is homeomorphic to $E^{1}$. Clearly, for $s_{0} \in \widetilde{F}, \pi_{1}\left(\widetilde{F}, s_{0}\right) \approx 0$. For $i=0, \pm 1, \pm 2, \ldots$, denote by $F_{i}$ a subset of $\widetilde{F}$ homeomorphic to $G$ such that $\widetilde{F}=\bigcup_{i=-\infty}^{\infty} F_{i-1} \cap F_{i} \neq \varnothing$ and for $k=0,1,2, \tau^{-1}\left(G^{k}\right)=\bigcup_{j=-\infty}^{\infty} F_{3 j+k}$.

Definition. Let $m$ be an integer. A homeomorphism $h: \widetilde{F} \rightarrow \widetilde{F}$ is said to be an $m$-shift homeomorphism if $h\left(F_{i}\right)=F_{i+m}$ for $i=0, \pm 1, \pm 2, \ldots$.

Definition. Let $m=0,1,2$. A homeomorphism $h: F \rightarrow F$ is said to be an $m$-shift homeomorphism if $h\left(G^{i}\right)=G^{(i+m) \bmod 3}$ for $i=0,1,2$.

Observe that for $i=0, \pm 1, \pm 2, \ldots, F_{i-1} \cap F_{i}$ is homeomorphic to $S^{2} \times S^{2}$.

From the properties of mapping cylinders and regular neighborhoods, it follows that the fourth homology group $H_{4}(\widetilde{F})$ is generated by $\left\{b_{i}\right\}_{i=-\infty}^{\infty}$, with relations $b_{i}=2 b_{i-1}$, where $b_{i}$ is obtained from the 4-manifold $F_{i-1} \cap F_{i}$ for $i=0, \pm 1, \pm 2, \ldots$. Moreover, by choosing an appropriate orientation of $F_{i-1} \cap F_{i}$, we may assume that the cycle representing $b_{i}$ has coefficient 1 on every simplex of $F_{i-1} \cap F_{i}$. Then, $b_{i}$ cannot be represented by a cycle with its carrier contained in $\bigcup_{j=i+1}^{\infty} F_{j}$ for $i=0, \pm 1, \pm 2, \ldots$. Also, note that if $h_{1}, h_{2}: \widetilde{F} \rightarrow \widetilde{F}\left(h_{1}, h_{2}: F \rightarrow F\right)$ are two isotopic $m_{1}$-shift and $m_{2}$-shift homeomorphisms, respectively, then $m_{1}=m_{2}$. 
Let $a$ be a generator of $H_{1}(K)$.

Lemma 13. If $h: F \rightarrow F$ is a homeomorphism, then $\left(\left.\bar{r} \circ h\right|_{K}\right)_{*}(a)=a$.

Proof. By [4, pp. 90-91] there exists a homeomorphism $\tilde{h}: \widetilde{F} \rightarrow \widetilde{F}$, and there exists a retraction $\tilde{r}: \widetilde{F} \rightarrow \tau^{-1}(K)$ such that the diagram

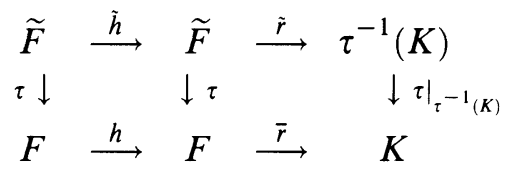

commutes.

Let $\left\{p_{n}\right\}_{n=1}^{\infty}$ be a sequence of points in $\widetilde{F}$. We will say that $\lim _{n \rightarrow \infty} p_{n}=\infty$ $\left[\lim _{n \rightarrow-\infty} p_{n}=-\infty\right]$ if for every integer $n_{0}$ almost all of the points $p_{n}$ belong to $\bigcup_{i=n_{0}}^{\infty} F_{i}\left[\bigcup_{i=-\infty}^{n_{0}} F_{i}\right]$. To prove Lemma 13, it is enough to show that if $\lim _{n \rightarrow \infty} p_{n}=\infty$, then $\lim _{n \rightarrow \infty} \tilde{h}\left(p_{n}\right)=\infty$ (hence $\lim _{n \rightarrow \infty} \tilde{r} \circ \tilde{h}\left(p_{n}\right)=\infty$ ) for every sequence $\left\{p_{n}\right\}_{n=1}^{\infty}$ contained in $\tau^{-1}(K)$.

There exist two sequences of positive integers $\left\{i_{n}\right\}_{n=1}^{\infty}$ and $\left\{j_{n}\right\}_{n=1}^{\infty}$ such that for every $n=1,2, \ldots$,

$$
\bigcup_{k=-i_{n}}^{i_{n}} F_{k} \subset \bigcup_{k=-j_{n}}^{j_{n}} \tilde{h}\left(F_{k}\right) \subset \bigcup_{k=-i_{n+1}}^{i_{n+1}} F_{k} .
$$

Note that if $n_{0} \geq 0$, then $\bigcup_{k=-n_{0}}^{n_{0}} F_{k}$ separates $\widetilde{F}$ between $\bigcup_{k=-\infty}^{-n_{0}-2} F_{k}$ and $\bigcup_{k=n_{0}+2}^{\infty} F_{k}$, and $\bigcup_{k=-n_{0}}^{n_{0}} \tilde{h}\left(F_{k}\right)$ separates $\tilde{F}$ between $\bigcup_{k=-\infty}^{-n_{0}-2} \tilde{h}\left(F_{k}\right)$ and $\bigcup_{k=n_{0}+2}^{\infty} \tilde{h}\left(F_{k}\right)$. Hence, there exists a strictly increasing sequence $\left\{i_{m}^{\prime}\right\}_{m=1}^{\infty}$, and a strictly increasing or decreasing sequence $\left\{j_{m}^{\prime}\right\}_{m=1}^{\infty}$ such that $\tilde{h}\left(F_{j_{m}^{\prime}}\right) \subset$ $\bigcup_{k=i_{m}^{\prime}}^{\infty} F_{k}$. If $\left\{j_{m}^{\prime}\right\}_{m=1}^{\infty}$ is strictly decreasing, then $\tilde{h}_{*}\left(b_{j_{0}^{\prime}}\right)$ can be represented by a cycle with its carrier in $\bigcup_{k=k_{0}}^{\infty} \tilde{h}\left(F_{k}\right)$ for some $k_{0}>j_{0}^{\prime}$, which is a contradiction. Hence, $\left\{j_{m}^{\prime}\right\}_{m=1}^{\infty}$ is strictly increasing, and if $\left\{p_{n}\right\}_{n=1}^{\infty}$ is a sequence with $\lim _{n \rightarrow \infty} p_{n}=\infty$, then $\lim _{n \rightarrow \infty} \tilde{h}\left(p_{n}\right)=\infty$.

Lemma 14. Let $X=S^{1} \times F$. Let $h: X \rightarrow X$ be a homeomorphism such that for every $\alpha \in S^{1}$ there exists an $\alpha^{\prime} \in S^{1}$ with $h(\{\alpha\} \times F)=\left\{\alpha^{\prime}\right\} \times F$. Let $\rho: \tilde{X} \rightarrow X$ be a covering map defined by setting $\widetilde{X}=S^{1} \times \widetilde{F}$ and $\rho=\operatorname{id}_{S^{1}} \times \tau$. Then there exists a map $\tilde{h}: \widetilde{X} \rightarrow \widetilde{X}$ such that the diagram

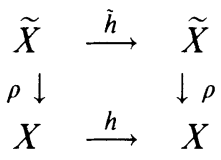

commutes.

Proof. As defined before, $S^{1}=\{\alpha: 0 \leq \alpha<2 \pi\}$. We may assume that both copies of $S^{1}$ appearing in $h: S^{1} \times F \rightarrow S^{1} \times F$ are parametrized in such a 
way that $h(\{\alpha\} \times F)=\{\alpha\} \times F$. Let $x_{0}=\left(0, s_{0}\right) \in X, y_{0}=\left(0, r_{0}\right) \in$ $X, \tilde{x}_{0}=\left(0, \tilde{s}_{0}\right) \in \widetilde{X}$, and $\tilde{y}_{0}=\left(0, \tilde{r}_{0}\right) \in \widetilde{X}$ be points such that $\rho\left(\tilde{x}_{0}\right)=$ $x_{0}, \rho\left(\tilde{y}_{0}\right)=y_{0}$, and $h\left(x_{0}\right)=y_{0}$. By [4, p. 90], it is enough to show that $(h \circ \rho)_{\#}\left(\pi_{1}\left(\tilde{X}, \tilde{x}_{0}\right)\right) \subset \rho_{\#}\left(\pi_{1}\left(\tilde{X}, \tilde{y}_{0}\right)\right)$. Let $f:[0,1] \rightarrow \tilde{X}$ be a loop defined by $f(\alpha)=\left(2 \pi \alpha \bmod 2 \pi, \tilde{s}_{0}\right)$; the loop $f$ represents a generator of $\pi_{1}\left(\tilde{X}, \tilde{x}_{0}\right)$.

For $0 \leq a \leq b \leq 2 \pi$, put

$$
X_{[a, b]}= \begin{cases}\{(\alpha, s) \in X: a \leq \alpha \leq b\} & \text { if } b \neq 2 \pi, \\ \{(\alpha, s) \in X: a \leq \alpha<b \text { or } \alpha=0\} & \text { if } b=2 \pi .\end{cases}
$$

Let $\tilde{X}_{[a, b]}=\rho^{-1}\left(X_{[a, b]}\right)$. Let $\tilde{h}_{1}: \widetilde{X}_{[0, \pi]} \rightarrow \widetilde{X}_{[0, \pi]}$ be the unique lifting of $\left.(h \circ \rho)\right|_{\widetilde{X}_{[0, \pi]}}$ with $\tilde{h}_{1}\left(\tilde{x}_{0}\right)=\tilde{y}_{0}$, and let $\tilde{h}_{2}: \widetilde{X}_{[\pi, 2 \pi]} \rightarrow \widetilde{X}_{[\pi, 2 \pi]}$ be the unique lifting of $\left.(h \circ \rho)\right|_{\widetilde{X}_{[\pi, 2 \pi]}}$ such that $\left.\tilde{h}_{2}\right|_{\widetilde{X}_{[\pi, \pi]}}=\left.\tilde{h}_{1}\right|_{\widetilde{X}_{[\pi, \pi]}}$. By [4, p. 86], there exists a path $\tilde{f}:[0,1] \rightarrow \widetilde{X}$ with $\tilde{f}(0)=\tilde{y}_{0}$ such that $\rho \circ \tilde{f}=h \circ \rho \circ f$. Let $i_{\alpha}: \widetilde{F} \rightarrow\{\alpha\} \times \widetilde{F}$ be the inclusion defined by $i_{\alpha}(s)=(\alpha, s)$, and let $\tilde{\pi}_{\widetilde{F}}: S^{1} \times \widetilde{F} \rightarrow \widetilde{F}$ be the projection. If $\tilde{f}(1) \neq \tilde{y}_{0}$, then $k=\pi_{\widetilde{F}} \circ \tilde{h}_{2}^{-1} \circ \tilde{h}_{1} \circ i_{0}: \widetilde{F} \rightarrow \widetilde{F}$ is a $3 n$-shift homeomorphism with $n \neq 0$. However, $\tilde{h}_{1}$ and $\tilde{h}_{2}$ yield an isotopy $H_{t}: \widetilde{F} \rightarrow \widetilde{F}$ defined by

$$
H_{t}=\pi_{\widetilde{F}} \circ \tilde{h}_{j}^{-1} \circ i_{2 \pi t} \circ \pi_{\widetilde{F}} \circ \tilde{h}_{1} \circ i_{0},
$$

where $j=1$ if $t \in\left[0, \frac{1}{2}\right)$ and $j=2$ if $t \in\left[\frac{1}{2}, 1\right]$. Hence, $H_{0}=\mathrm{id}_{\widetilde{F}}$ and $H_{1}=k$ are isotopic, which is a contradiction. Therefore, $\tilde{f}(1)=\tilde{y}_{0}$ and $(h \circ \rho)_{\#}\left(\pi_{1}\left(\tilde{X}, \tilde{x}_{0}\right)\right) \subset \rho_{\#}\left(\pi_{1}\left(\tilde{X}, \tilde{y}_{0}\right)\right)$.

Lemma 15. Let $U$ and $V$ be open connected subsets of $B$. Let $X=U \times F$, $Y=V \times F, \widetilde{X}=U \times \widetilde{F}$, and $\widetilde{Y}=V \times \widetilde{F}$. Let $\rho_{X}: \widetilde{X} \rightarrow X$ and $\rho_{Y}: \widetilde{Y} \rightarrow Y$ be covering maps defined by $\rho_{X}=\mathrm{id}_{U} \times \tau$ and $\rho_{Y}=\mathrm{id}_{V} \times \tau$ respectively. If $h: X \rightarrow Y$ is a homeomorphism, then there exists a map $\tilde{h}: \widetilde{X} \rightarrow \widetilde{Y}$ such that the diagram

commutes.

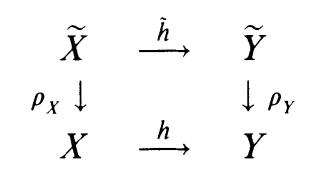

Proof. By Lemma 2, for every $u \in U$ there exists a $v \in V$ such that $h(\{u\} \times F)=\{v\} \times F$.

Let $x_{0} \in X, y_{0} \in Y, \tilde{x}_{0} \in \tilde{X}$, and $\tilde{y}_{0} \in \tilde{Y}$ be points such that $\rho_{X}\left(\tilde{x}_{0}\right)=x_{0}$, $\rho_{Y}\left(\tilde{y}_{0}\right)=y_{0}$, and $h\left(x_{0}\right)=y_{0}$. It is enough to show that if $f:[0,1] \rightarrow \widetilde{X}$ is a loop with $f(0)=f(1)=\tilde{x}_{0}$, then there exists a loop $\tilde{f}:[0,1] \rightarrow \tilde{Y}$ with $\tilde{f}(0)=\tilde{f}(1)=\tilde{y}_{0}$ such that $h \circ \rho_{X} \circ f=\rho_{Y} \circ \tilde{f}$. Without loss of generality, we may assume that $f([0,1]) \subset U \times\left\{\tilde{s}_{0}\right\}$, where $\tilde{x}_{0}=\left(u_{0}, \tilde{s}_{0}\right)$.

Let $t_{0}=0<t_{1}<\cdots<t_{m}=1$ be a sequence of points in [0,1], and let $f^{\prime}:[0,1] \rightarrow \tilde{X}$ be a loop such that $f^{\prime}([0,1]) \subset U \times\left\{\tilde{s}_{0}\right\}, f^{\prime}\left(t_{i}\right)=f\left(t_{i}\right)$ for $i=$ $0, \ldots, m,\left.f^{\prime}\right|_{[0,1]-\left\{t_{0}, \ldots, t_{m}\right\}}:[0,1]-\left\{t_{0}, \ldots, t_{m}\right\} \rightarrow f^{\prime}\left([0,1]-\left\{t_{0}, \ldots, t_{m}\right\}\right)$ 
is one-to-one, and for each $i=1, \ldots, m$, there is a $j=0,1,2$ such that $h \circ \rho_{X} \circ f^{\prime}\left(\left[t_{i-1}, t_{i}\right]\right) \cup h \circ \rho_{X} \circ f\left(\left[t_{i-1}, t_{i}\right]\right) \subset V \times\left(G^{j} \cup G^{(j+1) \bmod 3}\right)$.

If $P$ is a simple closed curve in $f^{\prime}([0,1])$, then $h \circ \rho_{X}(P)$ is a simple closed curve in $h \circ \rho_{X} \circ f^{\prime}([0,1])$. Let $\pi_{V}: V \times F \rightarrow V$ be the projection. Since for each $u \in U, h(\{u\}) \times F=\{v\} \times F$ for some $v \in V$, then $\pi_{V} \circ h \circ \rho_{X}(P)$ is a simple closed curve in $V$, and $h \circ\left(\rho_{X}(P) \times F\right)=\left(\pi_{V} \circ h \circ \rho_{X}(P)\right) \times F$. By Lemma 14 , for every $z \in \rho_{Y}^{-1} \circ h \circ \rho_{X}(P)$ there exists a simple closed curve $\widetilde{P} \subset \widetilde{Y}$ containing $z$ such that $\rho_{Y}(\widetilde{P})=h \circ \rho_{X}(P)$. Also, for $i=0, \ldots, m$, every loop whose image is the set $h \circ \rho_{X} \circ f^{\prime}\left(\left[t_{i-1}, t_{i}\right]\right) \cup h \circ \rho_{X} \circ f\left(\left[t_{i-1}, t_{i}\right]\right)$ lifts to a loop in $\tilde{F}$. Hence, there exists a loop $\tilde{f}:[0,1] \rightarrow \tilde{X}$ such that $h \circ \rho_{X} \circ f=\rho_{Y} \circ \tilde{f}$ and $\tilde{f}(0)=\tilde{f}(1)=\tilde{y}_{0}$.

Consider $C$ to be a subset of $D, C=\{(b, s) \in D: s \in L\}$. Let $s_{0}$ be the point of $L \cap G_{0}$. Consider $B$ to be a subset of $D, B=\left\{(b, s) \in D: s=s_{0}\right\}$. Let $R: D \rightarrow C$ be a retraction defined by $R(b, s)=(b, r(s))$, where $s \in G-G_{1}$. In this section, the notation $N_{p}$ is used for fibers of $D$ (homeomorphic to $F)$. Hence, if $p=\left(b_{1}, s_{1}\right)$, then $N_{p}=\left\{(b, s) \in D: b=b_{1}, b=f_{2}^{(3)}\left(b_{1}\right)\right.$, or $\left.b=f_{2}^{(6)}\left(b_{1}\right)\right\}$, where $f_{2}$ is the homeomorphism defined in $\S 2$. If $p \in C$, then the fiber of $C$ containing $p$, homeomorphic to $S^{1}$, is denoted by $K_{p}$, i.e., $K_{p}=N_{p} \cap C$.

Lemma 16. If $h: D \rightarrow D$ is a homeomorphism, then there exists an orientation preserving homeomorphism $\bar{h}: C \rightarrow C$ such that $\bar{h}(B)=B$ and such that for every $p$ and $q$ in $C$, if $h\left(N_{p}\right)=N_{q}$ then $\bar{h}\left(K_{p}\right)=K_{q}$.

Proof. Define a homeomorphism $\varphi: D \rightarrow D$ by $\varphi(b, s)=\left(f_{2}^{(3)}(b), s\right)$. Let $X=\left\{(r, \theta, z, \bar{x}, \bar{y}, \bar{z}, s) \in D: 0<\theta<\frac{2 \pi}{3}, \frac{2 \pi}{3}<\theta<\frac{4 \pi}{3}\right.$, or $\left.\frac{4 \pi}{3}<\theta<2 \pi\right\}$. Let $U$ be a component of $X \cap B$. Notice that $X$ is homeomorphic to $U \times F$ and the set $h(X) \cap B$ is not empty. Let $V$ be a component of $h(X) \cap B$.

We claim that $h(X)$ is homeomorphic to $V \times F$. Since $V$ is connected, if $V \cap \varphi(V) \neq \varnothing$, then $V$ is invariant under $\varphi$. Then, there exist a point $p_{0} \in V$ and an $\operatorname{arc} P_{0} \subset V$ joining $p_{0}$ and $\varphi\left(p_{0}\right)$ such that $P_{0} \cap \varphi\left(P_{0}\right)=\left\{p_{0}\right\}$. Hence $P_{0} \cup \varphi\left(P_{0}\right) \cup \varphi^{(2)}\left(P_{0}\right)$ is a simple closed curve contained in $V$. Consider the Cartesian product $P_{0} \times F$. Let $\pi_{F}: P_{0} \times F \rightarrow F$ be the projection, and let $i_{p}: F \rightarrow P_{0} \times F$ be the inclusion defined by $i_{p}(s)=(p, s)$ for $p \in P_{0}$. The set $Y=\bigcup_{p \in P_{0}} N_{p}$ is homeomorphic to $\left(P_{0} \times F\right) / k$, where $k:\left\{p_{0}\right\} \times F \rightarrow\left\{\varphi\left(p_{0}\right)\right\} \times F$ is a homeomorphism such that $\pi_{F} \circ k \circ i_{p_{0}}$ is a 1-shift homeomorphism.

The embedding $\left.h^{-1}\right|_{Y}: Y \rightarrow X$ preserves fibers, i.e., for every $p \in Y$, we have $\left(\left.h^{-1}\right|_{Y}\right)\left(N_{p}\right)=N_{q}$ for some $q \in X$. By an argument similar to that of the proof of Lemma $14, \pi_{F} \circ k \circ i_{p_{0}}$ is isotopic to the identity, which is a contradiction. Hence, $V \cap \varphi(V)=\varnothing$, and $\bigcup_{p \in V} N_{p}$ is homeomorphic to $V \times F$. Since $h(X)$ is connected, $h(X)=\bigcup_{p \in V} N_{p}$. Notice that for every $p \in V, V$ intersects each fiber $N_{p}$ at exactly one point, and there is a homeomorphism of $\bigcup_{p \in V} N_{p}$ 
onto $V \times F$ which takes each fiber $N_{p}$ onto some fiber $\{q\} \times F$.

Let $\omega: B \times \widetilde{F} \rightarrow D$ be a covering map such that $\omega(b, s)=(b, \tau(s))$ for $(b, s) \in B \times\left(F_{0}-F_{1}\right)$. By Lemma 15 , there exists a map $\tilde{h}_{U}: U \times \widetilde{F} \rightarrow B \times \widetilde{F}$ with $\tilde{h}(U \times \widetilde{F})=V \times \widetilde{F}$ and such that the diagram

$$
\begin{array}{ccc}
U \times \widetilde{F} & \stackrel{\tilde{h}_{U}}{\longrightarrow} & B \times \widetilde{F} \\
\left.\omega\right|_{U \times \widetilde{F}} \downarrow & & \downarrow \omega \\
X & \stackrel{\left.h\right|_{X}}{\longrightarrow} & D
\end{array}
$$

commutes.

For every $p \in \bar{U}$, the closure of $U$, there exists a neighborhood $W_{p}$ of $p$ in $B$ such that the set $\bigcup_{q \in W_{p}} N_{q}$ is homeomorphic to the Cartesian product $W_{p} \times F$. Furthermore, for every $p \in \bar{U}$, there exists a unique map $\tilde{h}_{p}: W_{p} \times \widetilde{F} \rightarrow$ $B \times \widetilde{F}$ such that the diagram

$$
\begin{aligned}
& W_{p} \times \widetilde{F} \stackrel{\tilde{h}_{p}}{\longrightarrow} B \times \widetilde{F} \\
& \left.\omega\right|_{W_{p} \times \widetilde{F}} \downarrow \quad \downarrow \omega \\
& \bigcup_{q \in W_{p}} N_{q} \stackrel{h \mid \bigcup_{q \in W_{p}}{ }^{N_{q}}}{\longrightarrow} D
\end{aligned}
$$

commutes, and $\left.\tilde{h}_{p}\right|_{\left(U \cap W_{p}\right) \times \widetilde{F}}=\left.\tilde{h}_{U}\right|_{\left(U \cap W_{p}\right) \times \widetilde{F}}$. Therefore there exists a map $\tilde{h}: \bar{U} \times \widetilde{F} \rightarrow B \times \widetilde{F}$ such that $\left.\tilde{h}\right|_{U \times \widetilde{F}}=\tilde{h}_{U}$. Note that $\tilde{h}(\bar{U} \times \widetilde{F})=\bar{V} \times \widetilde{F}$.

The set $\omega^{-1}(C)$ is homeomorphic to $B \times E^{1}$. Assume that $\omega^{-1}(C)=$ $B \times E^{1}$ and $\omega^{-1}(C) \cap(\bar{U} \times \widetilde{F})=\bar{U} \times E^{1}$. Let $(b, s) \in B \times E^{1}$, where $b=$ $(r, \theta, z, \bar{x}, \bar{y}, \bar{z})$. Let $i=0,1,2$. Assume that if $i \leq s \bmod 3<i+1$, then $\omega(b, s)=\left(b_{i}, s_{i}\right)$, where $b_{i}=\left(r, \theta+\frac{2 \pi i}{3}, z, \bar{x}, \bar{y}, \bar{z}\right)$, and $s_{i}=(s \bmod 3)-i$.

Let $\widetilde{R}: B \times \widetilde{F} \rightarrow B \times E^{1}$ be a retraction such that the diagram

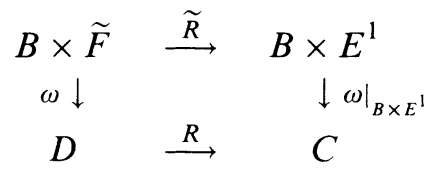

commutes. Note that if $p, \varphi(p) \in \bar{U}$ and $s \in E^{1}$, then

$$
\omega(p, s)=\omega(\varphi(p), s-1) .
$$

Hence, if $p, \varphi(p) \in \bar{U}, s \in E^{1}$, and $\widetilde{R} \circ \tilde{h}(p, s)=(q, r)$, then $\widetilde{R} \circ \tilde{h}(\varphi(p), s-1)$ $=(\varphi(q), r-1)$ or $\widetilde{R} \circ \tilde{h}(\varphi(p), s-1)=\left(\varphi^{(2)}(q), r+1\right)$. By Lemma 13, if $\widetilde{R} \circ \tilde{h}(p, s)=(q, r)$, then $\widetilde{R} \circ \tilde{h}(\varphi(p), s-1)=(\varphi(q), r-1)$ for $p, \varphi(p) \in \bar{U}$ and $s \in E^{1}$. Finally, if $(p, s) \in \bar{U} \times E^{1}$ is a point and $\widetilde{R} \circ \tilde{h}(p, s)=(q, r)$, then define $h^{\prime}: \bar{U} \times E^{1} \rightarrow \bar{V} \times E^{1}$ by $h^{\prime}(p, s)=(q, s)$. Let $\bar{h}: C \rightarrow C$ be such that the diagram

$$
\begin{array}{ccc}
\bar{U} \times E^{1} & \stackrel{h^{\prime}}{\longrightarrow} & \bar{V} \times E^{1} \\
\left.\omega\right|_{\bar{U} \times E^{1}} \downarrow & & \left.\downarrow \omega\right|_{\bar{V} \times E^{1}} \\
C & \stackrel{\bar{h}}{\longrightarrow} & C
\end{array}
$$


commutes. $\bar{h}$ is an orientation preserving homeomorphism, $\bar{h}(B)=B$, and if $h\left(N_{p}\right)=N_{q}$, then $\bar{h}\left(K_{p}\right)=K_{q}$ for $p, q \in C$.

Theorem. The continuum $D$ is homogeneous but not bihomogeneous.

Proof. By Lemma 4, $D$ is homogeneous. Let $p_{0}=\left(1,0,0,0,0,0, s_{0}\right)$ and $p_{1}=\left(1, \frac{2 \pi}{9}, 0,0,0,0, s_{0}\right)$. Suppose that there exists a homeomorphism $h: D \rightarrow D$ such that $h\left(p_{0}\right)=p_{1}$ and $h\left(p_{1}\right)=p_{0}$. Then, by Lemma 16 , there exists an orientation preserving homeomorphism $\bar{h}: C \rightarrow C$ such that $\bar{h}(B)=B$, $\bar{h}\left(K_{p_{0}}\right)=K_{p_{1}}$, and $\bar{h}\left(K_{p_{1}}\right)=K_{p_{0}}$. However, by Lemma $12, \bar{h}$ is orientation reversing. Therefore $D$ is not bihomogeneous.

\section{Problems}

Definition. A space $X$ is said to be semilocally bihomogeneous if for every $p$ there exists a neighborhood $U$ of $p$ such that for every $q \in U$ there exists a homeomorphism $h: X \rightarrow X$ with $h(p)=q$ and $h(q)=p$.

Remark 1 . The continuum $D$ constructed in this paper is semilocally bihomogeneous.

Problem 1. Does there exist a homogeneous continuum (locally connected continuum) which is not semilocally bihomogeneous?

Problem 2. Does there exist a homogenous, locally compact metric space (continuum) $X$ such that for no two points $p$ and $q$ in $X$ there exists a homeomorphism $h: X \rightarrow X$ with $h(p)=q$ and $h(q)=p$ ?

Remark 2. Cook's example (see [3]) of a homogeneous, nonbihomogeneous, locally compact metric space is of dimension 2 . The example constructed in this paper is of dimension 7.

Problem 3. What is the lowest dimension of a homogeneous, nonbihomogeneous, locally compact metric space (continuum)?

Remark 3. W. R. R. Transue points out that by his result of [8], Cook's example is embeddable in $E^{3}$.

Problem 4. Does there exist a homogeneous, nonbihomogeneous continuum embeddable in $E^{3}$ ?

Problem 5. Is every homogeneous, metric, absolute neighborhood retract bihomogeneous?

\section{REFERENCES}

1. R. D. Anderson, A characterization of the universal curve and a proof of its homogeneity, Ann. of Math. (2) 67 (1958), 313-324.

2. _ 1-dimensional continuous curves and a homogeneity theorem, Ann. of Math. (2) 68 (1958), 1-16. 
3. H. Cook, A locally compact, homogeneous metric space which is not bihomogeneous, Topology Proc. 11 (1986), 25-27.

4. S. T. Hu, Homotopy theory, Academic Press, New York, 1959.

5. K. Kuperberg, W. Kuperberg, and W. R. R. Transue, On the 2-homogeneity of Cartesian products, Fund. Math. 110 (1980), 131-134.

6. C. Kuratowski, Un probleme sur les ensembles homogènes, Fund. Math. 3 (1922), 14-19.

7. J. Nagata, Modern dimension theory, Bibl. Mat. 6 (1965).

8. W. R. R. Transue, On the hyperspace of subcontinua of the pseudoarc, Proc. Amer. Math. Soc. 18 (1967), 1074-1075.

9. G. S. Ungar, On all kinds of homogeneous spaces, Trans. Amer. Math. Soc. 212 (1975), 393-400.

10. D. van Danzig, Ueber topologish homogene Kontinua, Fund. Math. 15 (1930), 102-125.

Department of Foundations, Analysis and Topology, Auburn University, Auburn, Alabama 36849 